\title{
La percepción de las familias ante la enseñanza de segundas lenguas
}

\author{
The perception of families about the teaching of second languages
}

\author{
$\mathrm{M}^{\mathrm{a}}$ Ángeles Hernández Prados \\ $\mathrm{M}^{a}$ José Gambín Martínez \\ Ana Carmen Tolino Fernández-Henarejos \\ Universidad de Murcia
}

Recibido: 01/08/2017

Aceptado: 13/06/2018

\begin{abstract}
The knowledge of plurality of languages has become one of the most demanded professional competences, for this reason, the socio-educational relevance that has acquired the learning of second or third languages, in such a way that it has opened a new field of research approached mainly from a didactic approach. This article contains part of a research about teaching of second languages and penetrates in the analysis of school bilingualism. Specifically, it makes reference to the opinions of the families of secondary education students about the attitudes and degree of satisfaction that they present regarding the modality of bilingualism implanted in the schools. With a participant sample of 150 families, a descriptive-transversal study is developed. An ad hoc questionnaire composed of 45 and validated by experts was designed. The results show that families are satisfied with their children's English learning in bilingualism, however, those mothers who have a lower level of English have worse attitudes towards learning a second foreign language. These findings confirm the importance of teachers' relationships and implications with families and vice versa, promoting greater knowledge and satisfaction towards the bilingual modality

KEY WORDS: bilingual education, high school education, family, learning languages, foreign language

\section{RESUMEN}

El dominio de pluralidad de lenguas se ha convertido en una de las competencias profesionales más demandadas, de ahí, la relevancia socioeducativa que ha adquirido el aprendizaje de segundas o terceras lenguas, abriendo un nuevo campo de investigación que ha sido abordado principalmente desde un planteamiento didáctico. En este artículo se recoge parte de una investigación sobre la enseñanza de segundas lenguas que penetra en el análisis del bilingüismo escolar. En concreto se hace referencia a la opinión de las familias del alumnado de educación secundaria sobre las actitudes y grado de satisfacción que presentan ante la modalidad de bilingüismo implantada en los centros escolares. Con una muestra participante formada por 150 familias, se desarrolla un estudio descriptivo-transversal. Para ello se diseñó un cuestionario ad hoc compuesto por 45 ítems y validado por expertos. Los resultados señalan que las familias están satisfechas con el aprendizaje del inglés de sus hijos, sin embargo, aquellas madres que poseen un nivel de inglés menor tienen peores actitudes hacia el aprendizaje de una segunda lengua extranjera. Estos hallazgos confirman la importancia de las relaciones e implicaciones de los docentes con las familias y viceversa promoviendo un mayor conocimiento y satisfacción hacia la modalidad bilingüe.
\end{abstract}

PALABRAS CLAVE: educación bilingüe, educación secundaria, familia, actitudes, segunda lengua.

Dirección de correspondencia:

Ma Ángeles Hernández Prados, Universidad de Murcia. e-mail: mangeles@um.es. 


\section{Una aproximación a la educación bilingüe}

El estudio de la enseñanza de una segunda lengua (L2) en el contexto escolar cuenta con una larga tradición, aunque la definición de "Educación bilingüe" como el uso de dos o más lenguas en una parte del currículum escolar o en todas, descartando aquellos programas de enseñanza que solo incluyen a una lengua como asignatura, veía la luz de la mano de Cohen en el año 1975. A partir de este concepto, se promociona la proeficiencia bilingüe o plurilingüe cuando las lenguas se usan como un medio de instrucción mediante un currículo académico (Genesse, 2004). En esta misma línea expansiva, Mackey (1976) reconocía que el bilingüismo, lejos de ser un fenómeno excepcional exclusivo de los países bilingües, se encuentra en la mayor parte de población del globo terráqueo.

Los primeros estudios sobre el bilingüismo y el rendimiento escolar se centraban en evidenciar las dificultades de aprendizaje y el fracaso escolar que experimentaban especialmente el alumnado catalogado con bajo nivel sociocultural, y consideraban que el proceso de enseñanza-aprendizaje debía realizarse en la lengua materna de los escolares (Arnau, 1985). Sin embargo, tras más de 50 años, el concepto de bilingüismo nocivo e inseguro se ha ido volviendo más vulnerable tras comprobar las ventajas de la educación bilingüe en muchos campos (Morales, Calvo y Bialystok, 2013) y la relevancia socio-económica que acompañan a este tipo de aprendizajes.

Con la necesidad defendida de crear estados pluriculturales y plurilingües, los centros educativos de los diferentes países miembros de la Unión Europea se han visto envueltos en una gradual fase de adaptación a nivel multicultural y plurilingüe de sus sistemas educativos. Esta adopción ha sido posible gracias a la puesta en marcha del programa bilingüe llevado a cabo entre el Ministerio de Educación y Cultura y del Consejo Europeo en el año 2002. Ambas instituciones llegaron al acuerdo de que era necesario desarrollar y coordinar una serie de acciones educativas para alcanzar una economía competitiva y así como mejorar el dominio de al menos dos lenguas extranjeras desde una edad muy temprana (Real Decreto 1105/2014). Para ello se implantó un bilingüismo aditivo centrado en conocer el idioma, su estructura, vocabulario o sistema fonético del segundo idioma agregado al centro escolar sin que supusiera una amenaza para el desarrollo de la lengua materna (Cummins, 1990). Actualmente, la enseñanza bilingüe que se ofrece en nuestros centros educativos es una inmersión lingüística parcial y temprana (ORDEN ECI/1128/2006), que fomenta la diversidad lingüística a través del método natural de "baño de lengua" basado en la comunicación, la interacción y la priorización del código oral, lo que no implica la repetición, sino la articulación del mismo concepto de múltiples maneras diferentes. La finalidad última es la construcción de un "entorno de aprendizaje óptimo" donde se realicen las siguientes actuaciones básicas (Ellis, 1985): que el alumno reciba una gran cantidad de input en L2, que perciba la necesidad auténtica de comunicarse en L2, que tenga la oportunidad de oír y producir diferentes formas para las diferentes funciones de la lengua-objetivo, así como oportunidades para expresarse, confirmar y aclarar sus discursos y por último, que se exprese de manera libre, desinhibida en la L2 para experimentar nuevas formas en la L2.

Los estudios más recientes sobre bilingüismo se centran en averiguar los factores que favorecen el aprendizaje de una segunda lengua, las ventajas que conlleva en el rendimiento y desarrollo evolutivo de los menores, la búsqueda de nuevas metodologías más eficaces en la adquisición de esta competencia lingüística, especialmente desde un posicionamiento didáctico imperante, lo que implica que la mayoría de las veces se contempla la percepción del docente y del alumnado. Una breve revisión de los estudios realizados sobre los efectos que promueve el bilingüismo permite visualizar que se trata de una actividad cognitiva que afecta al cerebro produciendo cambios funcionales y estructurales en la regiones corticales que se dedican al procesamiento de la lengua y a la función ejecutiva (Crinion, 2006); dota de una mayor plasticidad neuronal y el sistema auditivo de las personas bilingües se vuelve altamente eficiente en el procesamiento automático del sonido codificando y percibiendo la sílaba de una manera más precisa que los monolingües (Krizman, Marian, Shook, Skoe y Kraus, 2012), y favorece el desempeño de tareas cognitivamente más exigentes de forma más eficiente (Morales, Calvo y Bialystok, 2013). El bilingüismo también facilita en adultos el aprendizaje de palabras nuevas, tal y como demuestra el estudio de Kaushanskaya y Marian (2009) en el que monolingües angloparlantes y bilingües 
primerizos de inglés-español e inglés-mandarín debían aprender palabras nuevas, comprobando que los dos grupos bilingües superaron al grupo monolingüe y demostrando que el bilingüismo facilita el aprendizaje de nuevos conceptos.

De igual modo, existe una amplia gama de programas para afianzar el aprendizaje de un segundo idioma en los que se resaltan diversidad de aspectos (Engle, 1975; Morales Gálvez, 2009). De forma general, la familia es considerada un factor determinante e ineludible que garantiza el proceso de enseñanza-aprendizaje (González-Pienda et al., 2002; Castejón, 2014), y más concretamente, la actitud y la percepción de los padres sobre el proceso de aprendizaje de un segundo idioma por parte de los hijos son determinantes. Esto se debe a que los hijos, desde niños ven proyectados su "yo" adolescentes a través del modelado, es decir, la imitación sobre la base de lo que observan en los adultos (Bandura y Walters, 1990). Se trata de un aprendizaje experiencial con altas dosis de credibilidad, perdurabilidad y fuertes lazos de emocionalidad. Es por ello que se espera que los padres sean un recurso más en la vida escolar de sus hijos y a su vez que colaboren con los profesores en la educación de sus hijos (Moreno Olivos, 2010).

Sin embargo, existen escasas investigaciones en las que se analice la percepción de las familias ante el bilingüismo, a pesar de que una buena percepción e implicación en la educación de sus hijos contribuye a una mejora del rendimiento académico (Martínez Segura, 2009). Por ello, es importante saber que un factor relevante para motivar o para desmotivar a un hijo es el ambiente familiar.

\subsection{La actitud de la familia ante la educación bilingüe}

El desarrollo y consolidación de los programas bilingües depende además del tiempo y calidad de la exposición al segundo idioma, de aspectos culturales y éticomorales que recaen en toda la comunidad educativa. Debe ser, en palabras de Lastra (2009), una construcción común de todos los agentes educativos para establecer objetivos compartidos y caminar en la misma dirección. Sin embargo, la responsabilidad educativa se diluye y se convierte en algo light, fruto de la divergencia entre el contexto escolar y el familiar, así como la dañina tendencia a focalizar la demanda en el papel del otro y no en las funciones propias. En este sentido, los resultados señalan que las familias deben proporcionar el aprendizaje a sus hijos, pero la responsabilidad del programa bilingüe es principalmente del profesorado y la administración.

En esta misma dirección apunta los resultados del estudio desarrollado por Rodríguez (2012) que avalan tanto la importancia del contexto sociocultural-familiar, de amistades - como del entorno del aula - el grupo de alumnos y especialmente de los profesores -para desarrollar la motivación y el ego lingǘstico del alumno que le empuje a participar más activamente en clase sin miedo a equivocarse, algo muy necesario del aprendizaje de otra lengua-cultura, pero conforme aumenta el nivel educativo del alumnado decrece la motivación hacia el aprendizaje del L2, como consecuencia del distanciamiento de las familias en la etapa de secundaria en adelante.

Si el aprendizaje de un idioma extranjero se produce por inmersión en los contextos cercanos, aprendiendo a valorar los idiomas como una forma de vida, la influencia del ambiente familiar como factor ineludible será determinante en el progreso escolar de los alumnos (González-Pienda et al., 2002; López Larrosa, 2002; Alonso Tapia, 2005). En este sentido, Torres (2010) realizó un estudio observacional en los hogares con la finalidad de analizar las estrategias comunicativas y discursivas que emplean, preocupaciones, factores, dudas y concepto que presentan los padres hacia el bilingüismo, concluyendo que a pesar de las particulares de cada familia existe una tendencia similar en estos aspectos en los padres de bilingüismo familiar en la toma de decisiones a la hora de fomentar el bilingüisimo. Esto se debe a que el apoyo que reciben los padres proviene de la propia experiencia, de la familia, de personas ajenas a la familia (profesores, pediatras, amigos, psicólogos,) y de "expertos" (doctores, libros) (King y Fogle, 2006; King y Mackey, 2007; Lanza, 2007; King, Fogle y Logan-Terry, 2008), condicionando la actitud de la familia hacia el bilingüismo.

La actitud entendida como un conjunto de componentes afectivos, conativos, cognitivos y evaluativos se manifiestan a través de creencias y opiniones sobre el proceso de enseñanzaaprendizaje de una LE (Manzaneda y Madrid, 1997). Las valoraciones positivas o negativas que se puedan hacer de la LE predisponen favorable o desfavorablemente al alumnado frente a las 


\section{$14 \mathrm{M}^{\mathrm{a}}$ Ángeles Hernández Prados, $\mathrm{M}^{\mathrm{a}}$ José Gambín Martínez \& Ana Carmen Tolino \\ Fernández-Henarejos}

situaciones de aprendizaje que se desarrollan en clase de LE (Uribe, Gutiérrez y Madrid, 2008), por lo tanto desempeñan "un papel fundamental en el aprendizaje de idiomas, ya que fortalecen la motivación de las personas para aprenderlos" (Masgoret Gardner, 2003, p.20). De modo que la percepción y valoración de las familias hacia el aprendizaje del segundo idioma hará que se sientan más motivados en clase de LE, y a la inversa (López García, 2009). Desafortunadamente, los padres continúan teniendo discrepancias y preocupaciones por la modalidad de aprendizaje bilingüe, incluso algunos de ellos muestran sus miedos a que el bilingüismo tenga efectos secundarios sobre sus hijos (Piller, 2001; King y Fogle, 2009; América, 2013). No obstante, las actitudes familiares hacia una educación bilingüe o monolingüe están determinadas por factores socio-psicológicos y socioeconómicos, de modo que la clase social media-alta, la posibilidad de interactuar socialmente fuera del núcleo familiar y la interacción dentro de la familia donde ambos cónyuges son un gran apoyo predisponen favorablemente hacia el bilinguismo (Hoffmann, 2014).

Partiendo de lo expuesto, se plantea el presente estudio con el propósito de averiguar la percepción de las familias hacia el bilingüismo de sus hijos estudiantes de Educación Secundaria Obligatoria. Concretamente, se pretende conocer la actitud que las familias presentan ante el aprendizaje de un idioma extranjero en la modalidad de bilingüismo y el grado de satisfacción de las familias respecto al proceso de enseñanza-aprendizaje del idioma extranjero en la modalidad de bilingüismo, así como analizar si existen diferencias significativas en la actitud y satisfacción de las familias hacia el bilingüismo en función de las variables predictoras contempladas en la investigación.

\section{Método}

Esta investigación se apoya en un diseño no experimental con una metodología descriptiva. Se trata de un estudio transversal multicéntrico que abarca todos los centros de secundaria del municipio murciano de Molina de Segura. Dado que la finalidad es describir, y no modificar, nos encontramos ante una investigación de tipo expost-facto, más específicamente ante un estudio correccional entre las variables contempladas, actitud y grado de satisfacción de las familias con el sistema bilingüe respecto a la variables independientes que son el índice socio económico, cultural, la nacionalidad, el tipo de centro, participación en el sistema bilingüe, idiomas estudiados dentro y fuera del centro y la persona que cumplimenta el cuestionario. Para la recogida y análisis de datos se ha empleado el cuestionario ad hoc que recoge la percepción de las familias de los alumnos de educación secundaria sobre la modalidad bilingüe a la que se adscriben sus hijos.

\subsection{Participantes}

Lamentablemente, los estudios dirigidos a familias suelen presentar una elevada tasa de mortandad en la muestra invitada. En esta ocasión un total de 560 familias cuyos hijos se encontraban escolarizados en el curso académico 2015-2016 en los centros de educación secundaria con modalidad bilingüe recibieron el instrumento de recogida de información, pero tan solo se obtuvieron 152 cuestionarios cumplimentados, de los cuales dos fueron anulados por estar parcialmente cumplimentados. Por tanto, la muestra resultante es de 150 familias, mostrándose una mayor participación de las madres en la cumplimentación del cuestionario $(67,3 \%)$. La muestra se caracteriza por estar representada mayoritariamente por familias de origen español (95\%), con hijos matriculados en centros públicos $(70 \%)$, con una alta ocupación laboral, especialmente en el sector masculino (Tabla 1), y un nivel de estudios de los padres y madres muy heterogéneo, situándose mayoritariamente en estudios medios (Tabla 2). En cuanto a la edad, casi tres cuartos de la muestra se sitúan en el intervalo de 41 a 50 años (tabla 3). 
Tabla 1

Situación laboral de los padres.

\begin{tabular}{lll}
\hline Progenitor & Situación laboral & Valores promedio \\
& Desempleado / jubilado & $7,00 \%$ \\
Padre & Parcial / temporal & $6,30 \%$ \\
& Completa & $86,70 \%$ \\
& Atención del hogar & $0 \%$ \\
& Desempleado / jubilado & $5,50 \%$ \\
Madre & Parcial / temporal & $13,80 \%$ \\
& Completa & $55,20 \%$ \\
& Atención del hogar & $25,50 \%$ \\
\hline
\end{tabular}

Tabla 2

Nivel de estudios de los padres.

\begin{tabular}{|c|c|c|}
\hline \multirow[t]{3}{*}{ Progenitor } & Nivel de estudios & Valores promedio \\
\hline & Sin estudios & $1,40 \%$ \\
\hline & Primaria & $18,19 \%$ \\
\hline \multirow[t]{5}{*}{ Padre } & Secundaria & $22,20 \%$ \\
\hline & Formación Profesional & $31,90 \%$ \\
\hline & Universitarios & $26,40 \%$ \\
\hline & Sin estudios & $0,70 \%$ \\
\hline & Primaria & $15,50 \%$ \\
\hline \multirow[t]{3}{*}{ Madre } & Secundaria & $20,90 \%$ \\
\hline & Formación Profesional & $29,70 \%$ \\
\hline & Universitarios & $33,10 \%$ \\
\hline
\end{tabular}

Tabla 3

Intervalo de edad de los padres.

\begin{tabular}{lll}
\hline Progenitor & Intervalos de edad & Valores promedio \\
& Menos de 40 & $5,00 \%$ \\
Padre & De 41 a 50 & $70,20 \%$ \\
& Más de 50 & $24,80 \%$ \\
Madre & Menos de 40 & $15,4 \%$ \\
& De 41 a 50 & $71,10 \%$ \\
\hline
\end{tabular}


Referente a la enseñanza reglada dentro del sistema bilingüe, los datos indican que un $96 \%$ de las familias que han participado tiene a su hijo/a dentro del sistema bilingüe de inglés del centro, frente a un 0,7 que no está dentro del programa bilingüe del centro. El inglés es el idioma estudiado dentro del centro por excelencia con un $99,3 \%$ y fuera del centro, un $45,2 \%$ lo estudia también fuera del centro. El francés es el segundo idioma más estudiado fuera del centro con un $29 \%$. Y un porcentaje de familias mucho menos, $3,2 \%$ pertenece a otros idiomas.

\subsection{Instrumento}

La recogida de datos se ha realizado con un cuestionario elaborado ad hoc, denominado "Percepción de las familias hacia el bilingüismo en Educación Secundaria Obligatoria", que se elaboró a partir de los indicadores obtenidos de la revisión de otros instrumentos. En dicho instrumento se distinguen dos partes. La primera se refiere a variables de identificación sociodemográficas de las familias (sexo, edad, nivel de estudios, situación laboral, titularidad del centro educativo, pertenencia del hijo al sistema bilingüe de centro, estudio de idiomas fuera del centro escolar, y nivel de competencia lingüística en una segunda lengua de la familia). La segunda está conformada por cuarenta y cinco ítems valorados por una escala de cuatro opciones $(1=$ nada, $2=$ poco, $3=$ bastante y 4=mucho) y estructurados en cuatro dimensiones: la actitud de las familias ante el aprendizaje de un idioma en la modalidad de bilingüismo (Rodríguez Pérez, 2012; King y Fogle, 2009); el grado de participación de las familias con todo lo relacionado con el bilingüismo en el ámbito escolar (Aira, 2013); el grado de implicación de las familias desde el hogar (Aira, 2013), y el grado de satisfacción de la modalidad bilingüe en la que estudian sus hijos (Ministerio de Educación, Cultura y deporte, 2001).

Para la validez de contenido del instrumento se desarrolló un proceso de valoración de interjueces. El panel de expertos estaba compuesto por 10 profesores, de los cuales 3 imparten docencia en educación superior y 7 en educación secundaria. Los criterios a valorar en cada ítem han sido: la adecuación, la pertinencia y la claridad, empleando una escala de 1 a 4 para la modalidad cuantitativa y una casilla por ítem para las propuestas de mejora. Los resultados indican que los jueces han valorado el cuestionario de manera muy positiva, obteniendo unas buenas valoraciones, casi todas con una nota media de 3 y 4 . La media más baja ha sido de 2,625. Igualmente se sugirieron cambios en algunos ítems estaban mal redactados y daban lugar a ambigüedad. Otra mejora era la inclusión de referencia de ambos sexos. Una vez adaptadas las propuestas de mejora indicadas, definitivamente se llevó a cabo la elaboración final del cuestionario.

Por otro lado, el análisis de fiabilidad arroja unos valores del Alfa de Cronbach de 0,774 para el conjunto total de los ítems, confirmando una buena consistencia interna por exceder el valor de 0,7 señalado por De Vellis (2003). En lo que respecta a la especificación de los valores de consistencia interna expresada por el coeficiente de Alfa de Cronbach en cada una de las dimensiones contempladas en el cuestionario, los resultados muestran una consistencia media para la actitud $(0,570)$ y la satisfacción de las familias ante el bilingüismo $(0,534)$.

Respecto a la validez de constructo del cuestionario se ha realizado un análisis de componentes principales, obteniendo una media de adecuación muestra de KMO (Kayser, Meyer y Olkin) de 0.550, en el mismo sentido, mediante la prueba de esfericidad de Bartlett se puede deducir que el modelo es significativo $(\mathrm{p}=.000)$. Específicamente, tanto en la dimensión de actitudes de las familias ante el bilingüismo, como en la de satisfacción de las familias hacia la modalidad bilingüe se han contemplado 3 componentes que explican el 50\% y el 60,51\% de la varianza (ver Tabla 4). 
Tabla 4

Varianza total explicada de las actitudes y la satisfacción familiar ante el bilingüismo

\begin{tabular}{|c|c|c|c|c|c|c|c|c|c|c|}
\hline \multirow{2}{*}{\multicolumn{2}{|c|}{ Componente }} & \multicolumn{3}{|c|}{ Autovalores iniciales } & \multicolumn{3}{|c|}{$\begin{array}{c}\text { Sumas de las saturaciones } \\
\text { al cuadrado de la } \\
\text { extracción }\end{array}$} & \multicolumn{3}{|c|}{$\begin{array}{l}\text { Suma de las saturaciones al } \\
\text { cuadrado de la rotación }\end{array}$} \\
\hline & & Total & $\begin{array}{l}\% \text { de la } \\
\text { varianza }\end{array}$ & $\begin{array}{c}\% \\
\text { acumul. }\end{array}$ & Total & $\begin{array}{l}\% \text { de la } \\
\text { varianza }\end{array}$ & $\begin{array}{c}\% \\
\text { acumul. }\end{array}$ & Total & $\begin{array}{l}\% \text { de la } \\
\text { varianza }\end{array}$ & $\begin{array}{c}\% \\
\text { acumul. }\end{array}$ \\
\hline & 1 & 2,49 & 22,63 & 22,64 & 2,49 & 22,64 & 22,63 & 2,36 & 21,495 & 21,49 \\
\hline \multirow[t]{3}{*}{ Actitud } & 2 & 1,83 & 16,67 & 39,31 & 1,83 & 16,67 & 39,31 & 1,58 & 14,412 & 35,91 \\
\hline & 3 & 1,19 & 10,88 & 50,19 & 1,19 & 10,88 & 50,19 & 1,57 & 14,279 & 50,19 \\
\hline & 1 & 3,70 & 33,67 & 33,67 & 3,70 & 33,67 & 33,67 & 2,63 & 23,898 & 23,89 \\
\hline \multirow[t]{2}{*}{ Satisfacción } & 2 & 1,63 & 14,81 & 48,49 & 1,63 & 14,82 & 48,49 & 2,16 & 19,670 & 43,57 \\
\hline & 3 & 1,32 & 12,03 & 60,52 & 1,32 & 12,03 & 60,52 & 1,86 & 16,95 & 60,52 \\
\hline
\end{tabular}

Método de extracción: Análisis de Componentes principales.

\subsection{Procedimiento}

En cuanto a la aplicación del instrumento para la recogida de datos, se llevó a cabo durante el mes de mayo del curso 2015 - 2016. Antes de la distribución del cuestionario, se procedió a la entrega en los centros educativos del consentimiento informado para hacer la recogida de la información dentro de unos límites legales y éticos. Se contactó con los equipos directivos y posteriormente con los tutores, ya que ellos actúan de mediadores con el alumnado e indirectamente a través de ellos con las familias. Tras la recogida se procedió al tratamiento de los datos a través del paquete estadístico SPSS v.19. El estudio estadístico de los datos se ha basado en un análisis estadístico descriptivo, mediante medidas de tendencia central y de dispersión de las variables para conocer la actitud y grado de satisfacción de las familias con la modalidad bilingüe, e inferencial a través de la prueba de correlación de Mann-Whitney y de Krushkal-Wallis, ya que la prueba de normalidad desveló que la muestra no sigue una distribución normal y por tanto hay que recurrir a estadística no paramétrica, con el objeto de conocer qué variables sociodemográficas de las familias inciden significativamente en la actitud y satisfacción hacia el bilingüismo de los hijos.

\section{Resultados}

En cuanto a la aplicación del instrumento para la recogida de datos, se llevó a cabo durante el mes de mayo del curso 2015 - 2016. Antes de la distribución del cuestionario, se procedió a la entrega en los centros educativos del consentimiento informado para hacer la recogida de la información dentro de unos límites legales y éticos. Se contactó con los equipos directivos y posteriormente con los tutores, ya que ellos actúan de mediadores con el alumnado e indirectamente a través de ellos con las familias.

Tras la recogida se procedió al tratamiento de los datos a través del paquete estadístico SPSS v.19. El estudio estadístico de los datos se ha basado en un análisis estadístico descriptivo, mediante medidas de tendencia central y de dispersión de las variables para conocer la actitud y grado de satisfacción de las familias con la modalidad bilingüe, e inferencial a través de la prueba de correlación de Mann-Whitney y de Krushkal-Wallis, ya que la prueba de normalidad desveló que la muestra no sigue una distribución normal y por tanto hay que recurrir a estadística no paramétrica, con el objeto 
de conocer qué variables sociodemográficas de las familias inciden significativamente en la actitud y satisfacción hacia el bilingüismo de los hijos.

Tabla 5

Estadísticos descriptivos totales

\begin{tabular}{lll}
\hline Actitudes hacia el bilingüismo & Media & Desviación típica \\
Actitud total & 2,6667 &, 34383 \\
Satisfacción total & 2,6468 &, 39644 \\
\hline
\end{tabular}

Para conocer la actitud que las presentan las familias ante el aprendizaje de un idioma extranjero en la modalidad de bilingüismo, hemos empleado unos ítems característicos de las ventajas que tiene aprender idiomas y por otra parte ítems de índole popular sobre el aprendizaje de idiomas. Y los resultados muestran (ver Tabla 6) que los padres consideran que aprender idiomas posibilita bastante el trabajar en otros países (media 3,8) así como aumentar las posibilidades de relacionarse (media 3,50), mostrando una actitud positiva hacia el bilingüismo. Sin embargo, creen que aprender idiomas no empobrece la lengua materna (media 1,33) y que el bilingüismo apenas provoca confusión a la hora de expresarse mezclando los dos idiomas (media 1,66), manifestando su desacuerdo con las actitudes negativas asociadas al aprendizaje de un idioma extranjero. También se produce una ruptura de los mitos que tradicionalmente acompañan al bilingüismo, pues las familias no creen que sólo se pueda aprender un idioma en su totalidad si se adquiere desde la infancia (media 1,83 ) y consideran que no entorpece el aprendizaje de otras materias (media 2,13)

Tabla 6

Estadísticos descriptivos de la actitud de las familias ante el aprendizaje de un idioma extranjero.

\begin{tabular}{|c|c|c|c|}
\hline & Actitudes hacia el bilingüismo & Media & $\begin{array}{l}\text { Desviación } \\
\text { típica }\end{array}$ \\
\hline \multirow{6}{*}{$\begin{array}{l}\text { Actitudes } \\
\text { positivas }\end{array}$} & $\begin{array}{l}\text { Aprender idiomas incrementa la capacidad } \\
\text { intelectual. }\end{array}$ & 3,41 & 667 \\
\hline & $\begin{array}{l}\text { Aprender idiomas incrementa las } \\
\text { posibilidades de relacionarse }\end{array}$ & 3,50 & ,740 \\
\hline & $\begin{array}{l}\text { Aprender idiomas posibilita para trabajar en } \\
\text { otros países. }\end{array}$ & 3,80 &, 418 \\
\hline & $\begin{array}{l}\text { Los padres tienen influencia en las actitudes } \\
\text { de sus hijos hacia el lenguaje. }\end{array}$ & 3,13 & 862 \\
\hline & $\begin{array}{l}\text { Aprender un idioma permite conocer otra } \\
\text { cultura }\end{array}$ & 3,37 & ,660 \\
\hline & $\begin{array}{l}\text { Para desarrollarbien el bilingüismo es muy } \\
\text { importante crear el hábito de la lectura. }\end{array}$ & 3,27 &, 730 \\
\hline \multirow{3}{*}{$\begin{array}{l}\text { Actitudes } \\
\text { negativas }\end{array}$} & $\begin{array}{l}\text { Si los padres no hablan bien, no deben } \\
\text { emplearla con sus hijos. }\end{array}$ & 2,11 & ,980 \\
\hline & $\begin{array}{l}\text { Aprender un idioma empobrece los } \\
\text { conocimientos de la lengua materna. }\end{array}$ & 1,13 &, 422 \\
\hline & $\begin{array}{l}\text { El bilingüismo provoca confusión a la hora } \\
\text { de expresarse mezclando ambos idiomas. }\end{array}$ & 1,66 &, 722 \\
\hline \multirow{2}{*}{ Mitos } & $\begin{array}{l}\text { Sólo se puede aprender un idioma en su } \\
\text { totalidad si se adquiere desdela infancia. }\end{array}$ & 1,83 &, 972 \\
\hline & $\begin{array}{l}\text { El bilingüismo no afecta al aprendizaje de } \\
\text { otras materias. }\end{array}$ & 2,13 & 1,189 \\
\hline & Actitud total & 2,6667 & ,34383 \\
\hline
\end{tabular}


En relación al grado de satisfacción de las familias respecto al proceso de aprendizajeenseñanza del inglés en la modalidad de bilingüismo (ver Tabla 7), podemos observamos que las familias se sienten satisfechas con el programa bilingüe de sus centros (media 2,75) y afirman que sus hijos comprenden bastante a sus profesores nativos de inglés (media 3,11), siendo a su vez lo bastante suficientes el número de horas de inglés por semana (media 2,96) y el nivel de inglés del material didáctico de su hijo $(1,96)$. Y por último, están poco de acuerdo en pensar que no hay calidad en la enseñanza del inglés en la E.S.O (media 2,13).

Tabla 7

Estadísticos descriptivos de la satisfacción de las familias ante el aprendizaje de un idioma extranjero.

\begin{tabular}{|c|c|c|c|}
\hline \multicolumn{2}{|c|}{$\begin{array}{l}\text { Consideraciones hacia el aprendizaje-enseñanza de inglés en la } \\
\text { modalidad bilingüe. }\end{array}$} & Media & $\begin{array}{l}\text { Desviación } \\
\text { típica }\end{array}$ \\
\hline \multirow{6}{*}{$\begin{array}{l}\text { Satisfacción respecto a } \\
\text { la modalidad bilingüe } \\
\text { de forma global }\end{array}$} & $\begin{array}{l}\text { Los profesores de idioma nativos son } \\
\text { mejores que los no nativos. }\end{array}$ & 2,61 & 1,117 \\
\hline & $\begin{array}{l}\text { Mi hijo/a ha mejorado en inglés gracias al } \\
\text { programa bilingüe del centro. }\end{array}$ & 2,62 & ,932 \\
\hline & $\begin{array}{l}\text { Me siento satisfecho con el programa } \\
\text { bilingüe del centro. }\end{array}$ & 2,75 & ,914 \\
\hline & $\begin{array}{l}\text { No hay calidad en la enseñanza del inglés en } \\
\text { la E.S.O }\end{array}$ & 2,13 & ,846 \\
\hline & $\begin{array}{l}\text { Considero que la cantidad de horas que mi } \\
\text { hijo da inglés a la semana son adecuadas. }\end{array}$ & 2,94 & ,921 \\
\hline & $\begin{array}{l}\text { A mi hijo/a le motiva estudiar inglés en el } \\
\text { centro. }\end{array}$ & 2,66 & ,898 \\
\hline \multirow{3}{*}{$\begin{array}{l}\text { Satisfacción respecto } \\
\text { al proceso enseñanza- } \\
\text { aprendizaje }\end{array}$} & $\begin{array}{l}\text { Mi hijo/a comprende a su profesor nativo de } \\
\text { inglés de conversación. }\end{array}$ & 3,11 & ,883 \\
\hline & $\begin{array}{l}\text { Mi hijo/a participa en las actividades } \\
\text { extraescolares culturales (teatro, } \\
\text { talleres...etc.) de inglés. }\end{array}$ & 2,83 & 1,132 \\
\hline & $\begin{array}{l}\text { Las actividades que realiza el departamento } \\
\text { de inglés en el centro son divertidas. }\end{array}$ & 2,55 & ,856 \\
\hline \multirow{3}{*}{$\begin{array}{l}\text { Satisfacción respecto a } \\
\text { la planificación }\end{array}$} & $\begin{array}{l}\text { El número de horas de inglés por semana } \\
\text { son suficientes. }\end{array}$ & 2,96 & ,866 \\
\hline & $\begin{array}{l}\text { Considero que el material didáctico que mi } \\
\text { hijo usa en inglés es de un nivel insuficiente. }\end{array}$ & 1,96 & 919 \\
\hline & Satisfacción total & 2,6468 & ,39644 \\
\hline
\end{tabular}

Para conocer la relación que existe entre las variables predictoras y de criterios, se han analizado si existen diferencias en lo que respecta a la actitud de las familias hacia el bilingüismo, la implicación hacia el bilingüismo en el contexto escolar y el hogar, y el grado de satisfacción de las familias con el proceso de enseñanza-aprendizaje del idioma extranjero. Para ello, se ha procedido a realizar un análisis inferencial a través de SPSS, recurriendo a estadística no paramétrica, ya que en función de la muestra y del instrumento empleado basado en escala de tipo ordinal, la forma indicada de proceder es esta.

De acuerdo a la Tabla 8 comprobamos que hay diferencias significativas entre la actitud ante el bilingüismo con el nivel de inglés por parte de la madre $(\mathrm{p}=0,001)$ cuyas significaciones serían entre mucho nivel de idioma y poco $(\mathrm{p}=0,17)$, por otra parte entre mucho y nada $(\mathrm{p}=0,01)$, y por último 
entre nada y poco $(p=0,004)$ donde se pueden observar grandes diferencias entre los valores medios de unos niveles de idioma a otros.

Tabla 8

Relación de la actitud con el nivel de inglés de la madre.

\begin{tabular}{lccc}
\hline \multicolumn{1}{c}{$\begin{array}{c}\text { Dominio del } \\
\text { inglés, madre }\end{array}$} & Sig. asintót & Nivel de inglés & Valores promedio \\
\hline &, 004 & Nada (1) & 50,45 \\
&, 001 & Poco (2) & 68,31 \\
$\begin{array}{l}\text { Dominio del inglés } \\
\text { de la madre } \\
(\mathrm{p}=0,001)\end{array}$ & & Nada (1) & 32,85 \\
&, 017 & Mucho (4) & 55,20 \\
& & Poco (2) & 31,13 \\
\end{tabular}

Por otra parte, nos encontramos con una cierta significación en la Tabla 9 entre la situación laboral de las madres y la actitud hacia el bilingüismo $(p=0,033)$, entre la situación laboral de contratación parcial/temporal y la contratación a modo completo $(\mathrm{p}=0,003)$ donde los valores promedio de la contratación temporal es inferior.

En la Tabla 10 observamos la significación entre la actitud sobre el bilingüismo de las familias con el hecho de que sus hijos estudien idiomas fuera del centro $(\mathrm{p}=0,037)$. Los idiomas más estudiados son el francés (media 15,76) y el inglés (media 9,64) donde el grado de significación de estos es $\mathrm{p}=0,035$.

Tabla 10

Relación de la actitud de los padres sobre el bilingüismo y el que los hijos estudien idiomas fuera del centro.

\begin{tabular}{lccc}
\hline Idiomas & Sig. asintót & $\begin{array}{c}\text { Idiomas fuera del } \\
\text { centro }\end{array}$ & Valores promedio \\
\hline $\begin{array}{l}\text { Idiomas fuera del } \\
\text { centro }(\mathrm{p}=0,037)\end{array}$ & \multirow{2}{*}{035} & Inglés (1) & 9,64 \\
\hline
\end{tabular}

De acuerdo con la Tabla 11 comprobamos que hay diferencias significativas entre la actitud ante el bilingüismo con el nivel de inglés por parte de la madre $(p=0,001)$ cuyas significaciones serían entre mucho nivel de idioma y poco $(p=0,17)$, por otra parte entre mucho y nada $(p=0,01)$, y por último entre nada y poco $(\mathrm{p}=0,004)$ donde se pueden observar grandes diferencias entre los valores medios de unos niveles de idioma a otros. 
Tabla 11

Relación de la actitud con el nivel de inglés de la madre.

\begin{tabular}{lccc}
\hline $\begin{array}{l}\text { Dominio del } \\
\text { inglés, madre }\end{array}$ & Sig. asintót & Nivel de inglés & Valores promedio \\
\hline &, 004 & Nada (1) & 50,45 \\
& & Poco (2) & 68,31 \\
Dominio del inglés &, 001 & Nada (1) & 32,85 \\
de la madre & & Mucho (4) & 55,20 \\
$(\mathrm{p}=0,001)$ & \multirow{2}{*}{, 017} & Poco (2) & 31,13 \\
& \multirow{2}{*}{0,55} & Mucho (4) & 46,75 \\
& & Bastante (3) & 11,76 \\
& & Mucho (4) & 17,80 \\
\hline
\end{tabular}

Respecto al alto grado de significación entre la satisfacción de las familias con el proceso de enseñanza-aprendizaje del inglés en la modalidad de bilingüismo y el nivel de inglés de la madre de la Tabla 12 ( $\mathrm{p}=0,008)$, se observan las siguientes significaciones: entre "nada" de nivel y "poco" $(p=0,005)$ y entre "nada" y "bastante" $(0,013)$, siendo los valores promedio de "nada" inferiores a sus respectivos, es decir, a menor nivel de inglés por parte de las madres, menor grado de satisfacción.

Tabla 12

Relación del grado de satisfacción de las familias del proceso de enseñanza-aprendizaje del inglés en la modalidad de bilingïismo con el nivel de inglés de la madre.

\begin{tabular}{lcll}
\hline $\begin{array}{l}\text { Dominio del } \\
\text { inglés, madre }\end{array}$ & Sig. asintót & Nivel de inglés & Valores promedio \\
\hline & \multirow{2}{*}{, 005} & Nada (1) & 50,68 \\
& & Poco (2) & 68,06 \\
& \multirow{2}{*}{, 013} & Nada (1) & 36,13 \\
& & Bastante (3) & 51,59 \\
Dominio del inglés & \multirow{2}{*}{, 099} & Nada (1) & 34,37 \\
de la madre & \multirow{2}{*}{ (201 } & Mucho (4) & 45,95 \\
& \multirow{2}{*}{$, 008)$} & Poco (2) & 35,26 \\
& \multirow{2}{*}{, 950} & Bastante (3) & 42,74 \\
& \multirow{2}{*}{, 266} & Poco (2) & 33,44 \\
& Mucho (4) & 33,85 \\
& Bastante (3) & 15,29 \\
& & Mucho (4) & 11,80 \\
\hline
\end{tabular}

A continuación, la Tabla 13 nos refleja una relación estadísticamente significativa $(\mathrm{p}=0,004)$ entre el grado de satisfacción de las familias ante la enseñanza-aprendizaje del inglés y el dominio de inglés del hermano. Las significaciones las encontramos entre nada y mucho $(\mathrm{p}=0,000)$ y entre ningún nivel y poco $(\mathrm{p}=0,002)$, siendo a favor cuando hay nivel de idioma por poco que sea. 
22 Ma Ángeles Hernández Prados, Ma José Gambín Martínez \& Ana Carmen Tolino

Fernández-Henarejos

Tabla 13

Relación del grado de satisfacción de las familias respecto al proceso de enseñanza-aprendizaje del inglés en la modalidad de bilingüismo con el dominio de inglés del hermano.

\begin{tabular}{lccc}
\hline $\begin{array}{l}\text { Dominio del } \\
\text { inglés, hermano }\end{array}$ & Sig. asintót & Nivel de inglés & Valores promedio \\
\hline & \multirow{2}{*}{, 002} & Nada (1) & 13,80 \\
Dominio del inglés & \multirow{2}{*}{, 008} & Poco (2) & 30,61 \\
del hermano & Nada (1) & 14,30 \\
$(\mathrm{p}=0,004)$ & \multirow{2}{*}{, 000} & Bastante (3) & 27,74 \\
& & Nada (1) & 8,30 \\
& & Mucho (4) & 21,33 \\
\hline
\end{tabular}

Por otra parte, en la Tabla 14 hay un alto grado de significación entre la percepción del rendimiento en inglés de los hijos de las familias encuestadas con la colaboración de los padres en las diferentes actividades que realiza el centro para las familias relacionadas con el aprendizaje y fomento del aprendizaje del inglés $(\mathrm{p}=0,001)$. Las relaciones de significación se dan entre aprobado y sobresaliente $(\mathrm{p}=0,000)$ y entre aprobado y notable $(\mathrm{p}=0,001)$ siendo a favor cuando el nivel es más elevado, en este caso el sobresaliente seguido del notable.

Tabla 14

Percepción de los padres del rendimiento de los hijos con la implicación en las tareas escolares relacionadas con el bilingüismo.

\begin{tabular}{llll}
$\begin{array}{l}\text { Pilinguismo. } \\
\text { Percepción del } \\
\text { rendimiento }\end{array}$ & Sig. asintót & $\begin{array}{l}\text { Nivel de inglés } \\
\text { percibido }\end{array}$ & Valores promedio \\
\hline & 0,36 & Suspenso (1) & 28,92 \\
& & Aprobado (2) & 18,38 \\
& \multirow{2}{*}{, 991} & Suspenso (1) & 34,92 \\
Percepción del & \multirow{2}{*}{, 646} & Notable (3) & 35,01 \\
rendimiento en & Suspenso (1) & 24,75 \\
inglés de los hijos & \multirow{2}{*}{, 001} & Sobresaliente (4) & 27,84 \\
(p=0,001) & \multirow{2}{*}{, 000} & Aprobado (2) & 35,56 \\
& \multirow{2}{*}{, 305} & Notable (3) & 55,28 \\
& Aprobado (2) & 28,56 \\
& Sobresaliente (4) & 49,55 \\
& Notable (3) & 53,28 \\
& Sobresaliente (4) & 59,57 \\
\hline
\end{tabular}

Acorde a la percepción que tienen los padres del rendimiento de sus hijos en inglés y el grado de satisfacción sobre la enseñanza bilingüe del centro, se observa una relación estadísticamente significativa $(\mathrm{p}=0,003)$ en la Tabla 15 donde la relación de significación se da entre suspenso y sobresaliente $(p=0,007)$ y a su vez entre suspenso y notable $(p=0,008)$. Esto quiere decir que los padres cuyos hijos tienen un mayor nivel de inglés percibido, más satisfechos se encuentran ellos. 
Tabla 15

Percepción de los padres del rendimiento de los hijos con su satisfacción sobre el proceso de enseñanza-aprendizaje del inglés en la modalidad de bilingüismo

\begin{tabular}{lcll}
$\begin{array}{l}\text { Percepción del } \\
\text { rendimiento }\end{array}$ & Sig. asintót & $\begin{array}{l}\text { Nivel de inglés } \\
\text { percibido }\end{array}$ & Valores promedio \\
\hline \multirow{2}{*}{0,51} & Suspenso (1) & 11,67 \\
& & $\begin{array}{l}\text { Aprobado (2) } \\
\text { Suspenso (1) }\end{array}$ & 21,52 \\
& \multirow{2}{*}{008} & Notable (3) & 14,25 \\
Percepción del & \multirow{2}{*}{, 007} & Suspenso (1) & 11,25 \\
rendimiento en & & Sobresaliente (4) & 29,53 \\
inglés de los hijos & \multirow{2}{*}{, 021} & Aprobado (2) & 39,47 \\
(p=0,003) & \multirow{2}{*}{, 016} & Notable (3) & 53,23 \\
& \multirow{2}{*}{, 483} & Aprobado (2) & 33,45 \\
& Sobresaliente (4) & 46,19 \\
& Notable (3) & 54,13 \\
& & Sobresaliente (4) & 58,45 \\
\hline
\end{tabular}

\section{Discusión y conclusiones}

Este trabajo permite una aproximación al bilingüismo desde la valoración y satisfacción de las familias cuyos hijos están recibiendo una educación bilingüe en los centros educativos de secundaria. Se parte del posicionamiento teórico de la educación sistémica en la que se debe atender a las relaciones entre instituciones participantes en la educación de los menores. De modo que cualquier cambio que se introduce en los centros educativos conlleva alteraciones en el conjunto de la comunidad educativa, de igual modo que los cambios familiares también alteran los procesos educativos que se desempeñan en el interior de los centros educativos. Parafraseando a Fuentes (2013) para evitar que la escuela se alce como única institución clave en la socialización de los niños, familia y escuela deben ser afines de forma natural, y establecer actuaciones comunes y coherentes sobre prácticas sociales, moral y valores a transmitir. Sin embargo, los estudios predominantes en el bilingüismo suelen primar la perspectiva del estudiante y del docente, incluso la didáctica, dejando en un segundo plano los estudios sobre las familias, sobretodo en el panorama español. Por ello, la primera conclusión que se extrae de este trabajo se desprende de la fundamentación y consulta de las fuentes, y consiste en reconocer la necesidad de incentivar estudios en este campo desde la perspectiva familiar, pues solo así se pueden detectar y poner de manifiesto tanto su percepción como las necesidades educativas de las familias derivadas de la implantación del bilingüismo en los centros escolares.

Uno de los puntos de partida de nuestro estudio fue conocer la percepción de las familias hacia el bilingüismo de sus hijos estudiantes de Educación Secundaria Obligatoria. Tras analizar los resultados expuestos respecto esta variable, podemos concluir entre otros aspectos, que predomina una buena actitud y un buen nivel de inglés por parte de los padres, que da lugar a una mayor satisfacción por su parte hacia el bilingüismo y por parte de los alumnos se obtienen mejores notas académicas. Este hecho pone fin a las preocupaciones de los padres por la modalidad de bilingüismo estudiadas en investigaciones precedentes como son la posibilidad de retrasos y desórdenes en la adquisición lingüística de sus hijos y así como el hecho de que el bilingüismo acabase provocándoles determinadas confusiones y acabasen mezclándolos (King y Fogle, 2009).

Más específicamente, en lo que respecta a las actitudes, los estudios realizados por Masgoret Gardner (1999) nos confirman que las actitudes juegan un papel fundamental en el aprendizaje de idiomas, ya que fortalecen la motivación para aprenderlos. Esto se debe a que las actitudes son un conjunto de componentes afectivos, conativos, cognitivos y evaluativos manifestados a través de creencias y opiniones sobre el aprendizaje de una lengua (Manzaneda y Madrid, 1997). La presente 


\section{$24 \mathrm{M}^{\mathrm{a}}$ Ángeles Hernández Prados , Ma José Gambín Martínez \& Ana Carmen Tolino \\ Fernández-Henarejos}

investigación se ha planteado como objetivo específico conocer la actitud que las familias presentan ante el aprendizaje de un idioma extranjero en la modalidad de bilingüismo, comprobado que la actitud de los padres y madres de los alumnos de educación secundaria hacia el bilingüismo es positiva, pues predomina una disposición favorable hacia el aprendizaje de idioma tanto del francés como del inglés fuera del centro, siendo las madres las que muestran mejores actitudes, especialmente las que tienen un mayor nivel de inglés. Al respecto, Cirugeda y Campillo (2016) señalan el incremento de alumnos que acuden a actividades extraescolares para la adquisición y refuerzo de la lengua extranjera, así como el visionado de vídeos en versión original, como prácticas habituales en las unidades familiares, lo que denota una mejora de la actitud de las familias. En general, la actitud positiva de las familias traducida en una mayor participación e implicación hacia la educación de sus hijos tiene una gran influencia en el éxito académico y global de todos los estudiantes (Epstein y Sanders, 2000, Consejo Escolar del Estado, 2014), del mismo modo, el efecto de un alto nivel participación y una actitud positiva de padres y madres en el rendimiento en los programas de idiomas extranjeros correlacionan significativamente con el nivel de aprendizaje de sus hijos (Hosseinpour, Sherkatolabbasi y Yarahmadi, 2015).

Teniendo en cuenta la importancia de una buena actitud en las familias para el aprendizaje de las lenguas extranjeras (Rosenbusch, 1987) y para predecir el nivel de éxito de los niños en el logro lingǘstico (Baker, 1992), los resultados obtenidos nos permiten concluir que se debe aprovechar la buena disposición de las familias hacia la adquisición de un segundo idioma, tanto dentro como fuera del sistema escolar, para establecer protocolos de acogida de los padres a los centros bilingües, fomento de buenas prácticas en el contexto familiar, asesoramiento respecto a los centros externos de enseñanza de idiomas, entre otras actuaciones, todo ello en mejora de la calidad de la educación que reciben los niños y de un mayor logro de la competencia lingüística.

Además de conocer las actitudes de las familias hacia el bilingüismo, se estudió el grado de satisfacción de las familias respecto al proceso de enseñanza-aprendizaje del idioma extranjero en la modalidad de bilingüismo. Tras el análisis de los resultados obtenidos se ha comprobado que el grado de satisfacción en la modalidad de aprendizaje bilingüe de las familias se encuentra estrechamente ligado al nivel de inglés de las madres, de modo que a mayor nivel de inglés por parte de la madre mayor grado de satisfacción. Por otra parte, las familias en su conjunto, se sienten satisfechas con el programa bilingüe del centro de su hijo y disponen a su vez de otro hijo estudiando inglés con "poco" o "ningún" nivel. Como en otros estudios, más específicamente el realizado por Parkes y Ruth (2011), los datos desvelan que a pesar de las preocupaciones por la modalidad bilingüe en lo que respecta al desarrollo del lenguaje y alfabetización, las habilidades matemáticas, la comunicación entre la familia y la escuela, y las dificultades académicas asociadas al rendimiento escolar, los padres se sienten mayoritaria y altamente satisfechos con el programa bilingüe presentado en los centros escolares de sus hijos, especialmente aquellos que se implican en el desarrollo educativo desde el hogar, aunque también es posible que los padres menos satisfechos no participaran en el estudio. De todo ello se concluye la necesidad de promover una mayor implicación de las familias en los procesos de enseñanza-aprendizaje del idioma extranjero, favoreciendo de este modo una mejora de la satisfacción hacia el bilingüismo.

Para finalizar, el estudio realizado también pone de manifiesto la existencia de un alto grado de significación entre la percepción de padres y madres respecto al rendimiento académico en inglés de sus hijos y la satisfacción sobre el proceso de enseñanza-aprendizaje del inglés en la modalidad de bilingüismo. Más concretamente, se manifiesta que la percepción de las familias sobre el rendimiento en inglés de sus hijos es de notable y sobresaliente. Por lo tanto, cuanto más satisfechas están las familias con el aprendizaje de inglés en la modalidad de bilingüismo, mayor es el nivel de inglés que perciben de sus hijos. 
Referencias bibliográficas

Aira, A. (2013). El bilingüismo y sus efectos en estudiantes de nivel secundario. Universidad de Gotemburgo, Gotemburgo. Recuperado de https://gupea.ub.gu.se/bitstream/2077/35209/1/gupea_2077_35209_1.pdf

Arnau, J. (1985). Educación en la segunda lenguas y rendimiento escolar: una revisión de la problemática general. En M. Siguan (coord.) Enseñanza en dos lenguas y resultados escolares (7-20). Barcelona, Edicions Universitat Barcelona.

Bandura, A. \& Walters, R . (1990). Aprendizaje social y desarrollo de la personalidad. Madrid, Alianza Editorial.

Baker, C. (1992), Attitudes and Language. Clevedon, USA: Multilingual Matters

Castejón, J.L. (2014). Aprendiraje y rendimiento académico. España, Editorial Club Universitario.

Cirugeda López, I. \& Campillo López, R. M. (2016) El método sistemático-sintético de lectoescritura Phonics como herramienta para la adquisición de la fonética inglesa. Revista Fuentes, 18 (2), 183-195.

Cohen, A. (1975). A sociolinguistic approach to bilingual education: experiments in the American Southwest. Newbury House Publishers.

Crinion, J., Turner, R., Grogan, A., Hanakawa, T., Noppeney, U., Devlin, J. T., Aso, T,, Urayama, S., Fukuyama, H., Stockton, K., Usui, K., Green, D.W., \& Price, C.J.. (2006). Language control in the bilingual brain. Science, 312(5779), 1537-1540.

Cummins, J. (1990). The Academic, Intellectual, and Linguistic Benefits of Bilingualism. So you want your child to learn French, 89-95. Canadian Parents for French. Recuperado de: htto://www.cpfcalEnglishlParentslbenefitsbilingualism.htm

De Vellis, R.F. (2003). Scale development: Theory and applications ( $2^{a}$ ed.). Thousand Oaks, CA: Sage.

Engle, P. (1975). Language Medium in Early School Years for Minority Language Groups. Review of Educational Research, 40, 2, 283-325.

Epstein, J. L., \& Sanders, M. G. (2000). Connecting home, school, and community: New directions for social research. In M. T. Hallinan (Ed.), Handbook of the sociology of education (pp. 285-306). New York, NY: Kluwer Academic/Plenum Publishers.

Ellis, R. (1985). Understanding second language acquisition. Oxford University Press.

Fuentes, S. (2013). School choices: moral and distinction in the relation family-school. Cuadernos de Pesquisa, 43 (149), 682-703

Genesee, F. (2004). What do we know about bilingual education for majority language students? In T. K. Bhaktia, \& W. Richie (Eds.), Handbook of Bilingualism and Multilingualism (547-576). Malden, MA: Blackwell.

González-Pienda, J.A., Núñez Pérez, J.C., González-Pumariega, S., Alvarez, L., Roces, C. \& García, M. (2002). A Structural Equation model of Parental Involment, Motivational and Aptitudinal Characteristics, and Academic Achievement. The Journal of Experimental Education, 70 (3), 257287.

Hoffmann, C. (2014). An introduction to bilingualism. Routledge: Taylor \& Francis.

Hosseinpour, V., Sherkatolabbasi, M. \& Yarahmadi, M. (2015). The impact of parents' involvement in and attitude toward their children's foreign language programs for learning English. International Journal of Applied Linguistics and English Literature, 4(4), 175-185.

Lanza, E. (2007). Multilingualism and the family. En P. Auer y Li Wei (Eds.), Handbook of multilingualism and multilingual communication (pp. 45-68). New York: Mouton of Gruyter.

Uribe, D., Gutiérrez, J. \& Madrid, D. (2008). Las actitudes del alumnado hacia el aprendizaje del inglés como idioma extranjero: estudio de una muestra en el sur de España. Porta Linguarum, $\mathrm{n}^{\mathrm{o}} 10, \mathrm{pp} .85-100$.

Rosenbusch, M. (1987). Second language learning in Young children. Lowa State University. Recuperado de: http://lib.dr.iastate.edu/rtd/8581/ 
Kaushanskaya, M. \& Marian, V. (2009). The bilingual advantage in novel word learning. Psychonomic Bulletin \& Review, 18(4), 705-710.

King, K. \& Fogle, L. (2006). Raising bilingual children: Common parental concerns and current research. Center for Applied Linguistics, 26, 17-29.

King, K. \& Fogle, L. (2009). La crianza de niños bilingües: preocupaciones comunes de los padres y las investigaciones actuales. Revista CALdigest, febrero, 1 - 4.

King, K. A., Fogle, L. \& Logan-Terry, A. (2008) Family language policy. Language and linguistics compass, 2(5), 907-922.

King, K. \& Mackey, A. 2007. The bilingual edge. New York: Harper Collins.

Krizman, J., Marian, V., Shook, A., Skoe, E. \& Kraus, N. (2012). Subcortical encoding of sound is enbanced in bilinguals and relates to execute function advantages. San Francisco: Keck Center for Integrative Neuroscience.

Larrosa, S. \& Rodríguez-Arias, J. L. (2002) Factores de riesgo y de protección en el consumo de drogas y la conducta antisocial en adolescentes y jóvenes españoles (Tesis doctoral). LA Coruña: Universidad de Coruña. Recuperado de https://dialnet.unirioja.es/descarga/articulo/3974485.pdf

Lastra, S. (2009). An Understanding of the Concept and Conditions of Bilingualism: A Study in an EFL Setting. How Journal, 16 , pp. 167 - 187.

López García, Á. 2009. La lengua común en la España plurilingüe. Madrid: Francfort del Meno, Iberoamericana y Vervuert.

Mackey, W.-F (1976). Bilinguisme et contact des langues. París, Editions Klincksieck.

Manzaneda, F. \& D. Madrid (1997). Actitudes y motivación en la clase de inglés ( $1^{\circ}$ de BUP), en E. A. Adams, A. Bueno y G. Tejada (eds) (1997) Francisco Manzaneda Oneto"In Memoriam" (153171). Universidad de Jaén: Servicio de Publicaciones.

Masgoret, A. M. \& Gardner, R. C. (2003). Attitudes, motivation and Second Language Learning: A meta-analysis of studies conducted by Gardner and Associates. Language Learning, 53, 1: 123163.

Ministerio de Educación, Cultura y deporte, (MECD). (2012). La enseñanza de las lenguas extranjeras en el sistema educativo español. Colección Eurydice España-Redie.

Morales Gálvez, C. (2009) La enseñanza de lenguas extranjeras en la Unión Europea. Educación y future: revista de investigación aplicada y experiencias educativas (20), 17 - 30.

Morales, J., Calvo, A., \& Bialystok, E. (2013). Working memory development in monolingual and bilingual children. Journal of experimental child psychology, 114(2), 187-202.

Moreno Olivos, T. (2010) La relación familia-escuela en secundaria: algunas razones del fracaso escolar. Profesorado, Revista de Curriculum y Formación del Profesorado, 14 (2). Recuperado de http://www.ugr.es/ recfpro/rev142COL3.pdf

Orden ECI 1128/2006, de 6 de abril, por la que se desarrolla el Real Decreto 717/2005, de 20 de junio, por el que se regula la ordenación de las enseñanzas en los centros docentes acogidos al convenio entre el Ministerio de Educación y Ciencia y The British Council.

Parkes, J. \& Ruth, T. (2011). How satisfied are parents of students in dual language education programs? International Journal of Bilingual Education and Bilingualism, 14(6), 701-718.

Pavlenko, A., Blackledge, A., Piller, I. \& Teutsch-Dwyer, M. (2004). Multilingualism, second language learning, and gender. Studies in second language acquisition, Vol.26 (1). p.125.

Piller, I. (2001) Who, if anyone, is a native speaker? Anglistik. Mitteilungen des Verbandes Deutscher Anglisten, 12 (2), p.109-121

Real Decreto 1105/2014, de 26 de diciembre, por el que se establece el currículo básico de la Educación Secundaria Obligatoria y del Bachillerato.

Rodríguez-Pérez, N. (2012). Causas que intervienen en la motivación del alumno en la enseñanzaaprendizaje de idiomas: el pensamiento del profesor. Didáctica. Lengua y Literatura, 24, 381409.

Tapia, J. (2005). Motivar en la escuela, motivar en la familia. Madrid: Ediciones Morata.

Torres, J. (2010). Aspectos de la planificación lingüistica de familias bilingües español/inglés en Sevilla. Sevilla: Universidad de Sevilla. 


\section{Para citar este artículo}

Hernández Prados, M. A., Gambín Martínez, M.J. \& Tolino Hernández-Henarejos, A. C. (2018). La percepción de las familias ante la enseñanza de segundas lenguas. Revista Fuentes, 20(1), 11-27. [Fecha de consulta: dd/mm/aa].

doi: http://dx.doi.org/10.12795/revistafuentes.2018.v20.i1.01 